\title{
Some results on chromaticity of quasi-linear paths and cycles
}

\author{
Ioan Tomescu \\ Faculty of Mathematics and Computer Science \\ University of Bucharest \\ Bucharest, Romania \\ and \\ Abdus Salam School of Mathematical Sciences \\ GC University, Lahore, Pakistan \\ ioan@fmi.unibuc.ro \\ Submitted: Feb 7, 2012; Accepted: May 21, 2012; Published: May 31, 2012 \\ Mathematics Subject Classifications: 05C15, 05C65
}

\begin{abstract}
Let $r \geqslant 1$ be an integer. An $h$-hypergraph $H$ is said to be $r$-quasi-linear (linear for $r=1$ ) if any two edges of $H$ intersect in 0 or $r$ vertices. In this paper it is shown that $r$-quasi-linear paths $P_{m}^{h, r}$ of length $m \geqslant 1$ and cycles $C_{m}^{h, r}$ of length $m \geqslant 3$ are chromatically unique in the set of $h$-uniform $r$-quasi-linear hypergraphs provided $r \geqslant 2$ and $h \geqslant 3 r-1$.
\end{abstract}

Keywords: quasi-linear hypergraph; sunflower hypergraph; quasi-linear path; quasi-linear cycle; chromatic polynomial; chromatic uniqueness; potential function

\section{$1 \quad$ Notation and preliminary results}

A simple hypergraph $H=(V, \mathcal{E})$, with order $n=|V|$ and size $m=|\mathcal{E}|$, consists of a vertex-set $V(H)=V$ and an edge-set $E(H)=\mathcal{E}$, where $E \subseteq V$ and $|E| \geqslant 2$ for each edge $E$ in $\mathcal{E}$. $H$ is $h$-uniform, or is an $h$-hypergraph, if $|E|=h$ for each $E$ in $\mathcal{E}$ and $H$ is linear if no two edges intersect in more than one vertex [1]. $H$ is said to be antilinear if for every two edges $E, F$ of $H$ we have $|E \cap F| \neq 1$. Let $r \geqslant 1$ and $h \geqslant 2 r+1$. $H$ is said to be $r$-quasi-linear (or shortly quasi-linear) [13] if any two edges intersect in 0 or $r$ vertices. Examples of quasi-linear hypergraphs are $t$-stars [5, 8], also called sunflower hypergraphs $[7,11,12]$. We say that a hypergraph $S$ is a $t$-star with kernel $K$ where $K \subseteq V(S)$ and $t \geqslant 1$ if $S$ has exactly $t$ edges and $e \cap e^{\prime}=K$ for all distinct edges $e$ and $e^{\prime}$ of $S$. A system of $t$ pairwise disjoint edges (matching) is a $t$-star with empty kernel. In [12] a 
sunflower hypergraph was denoted by $S H(n, p, h)$; it is an $h$-hypergraph having a kernel of cardinality $h-p, n$ vertices and $k$ edges, where $n=h+(k-1) p$ and $1 \leqslant p \leqslant h-1$. A hypergraph for which no edge is a subset of any other is called Sperner. Two vertices $u, v \in V(H)$ belong to the same component if there are vertices $x_{0}=u, x_{1}, \ldots, x_{k}=v$ and edges $E_{1}, \ldots, E_{k}$ of $H$ such that $x_{i-1}, x_{i} \in E_{i}$ for each $i(1 \leqslant i \leqslant k)$ [1]. $H$ is said to be connected if it has only one component. An $h$-uniform hypertree is a connected linear $h$-hypergraph without cycles. We shall define two classes of quasi-linear uniform hypergraphs called quasi-linear elementary paths and quasi-linear elementary cycles and denoted by $P_{m}^{h, r}$ and $C_{m}^{h, r}$, respectively, as follows: $P_{m}^{h, r}$ consists of $m$ edges $E_{1}, \ldots, E_{m}$ such that $\left|E_{1}\right|=\ldots=\left|E_{m}\right|=h,\left|E_{k} \cap E_{l}\right|=r$ if $\{k, l\}=\{i, i+1\}$ for any $1 \leqslant i \leqslant m-1$ and 0 otherwise. Cycles $C_{m}^{h, r}$ are defined analogously, by also imposing $\left|E_{m} \cap E_{1}\right|=r$.

If $\lambda \in \mathbb{N}$, a $\lambda$-coloring of a hypergraph $H$ is a function $f: V(H) \rightarrow\{1, \ldots, \lambda\}$ such that for each edge $E$ of $H$ there exist $x, y$ in $E$ for which $f(x) \neq f(y)$. The number of $\lambda$ colorings of $H$ is given by a polynomial $P(H, \lambda)$ of degree $|V(H)|$ in $\lambda$, called the chromatic polynomial of $H . P(H, \lambda)$ can be obtained applying inclusion-exclusion principle, in the same way as for graphs, getting the following formula:

$$
P(H, \lambda)=\sum_{W \subseteq E(H)}(-1)^{|W|} \lambda^{c(W)},
$$

where $c(W)$ denotes the number of components of the spanning subhypergraph induced by the edges from $W$. By rearranging terms in (1) we obtain that if $H$ has order $n$ then $P(H, \lambda)=\lambda^{n}+a_{n-1} \lambda^{n-1}+\ldots+a_{1} \lambda$, where

$$
a_{i}=\sum_{j \geqslant 0}(-1)^{j} N(i, j)
$$

and $N(i, j)$ denotes the number of spanning subhypergraphs of $H$ with $n$ vertices, $i$ components and $j$ edges [10].

All $h$-uniform hypertrees have the same chromatic polynomial.

Lemma 1. [6]. If $T_{k}^{h}$ is any h-uniform hypertree with $k$ edges, then

$$
P\left(T_{k}^{h}, \lambda\right)=\lambda\left(\lambda^{h-1}-1\right)^{k} .
$$

Two hypergraphs $H$ and $G$ are said to be chromatically equivalent or $\chi$-equivalent, written $H \sim G$, if $P(H, \lambda)=P(G, \lambda)$. Let us restrict ourselves to the class of Sperner hypergraphs. A simple hypergraph $H$ is said to be chromatically unique if $H$ is isomorphic to $H^{\prime}$ for every simple hypergraph $H^{\prime}$ such that $H^{\prime} \sim H$; that is, the structure of $H$ is uniquely determined up to isomorphism by its chromatic polynomial. The notion of $\chi$ unique graphs was first introduced and studied by Chao and Whitehead [4] (see also [9]). It is clear that all $h$-hypergraphs are Sperner. The notion of $\chi$-uniqueness in the class of $h$ hypergraphs may be defined as follows: An $h$-hypergraph $H$ is said to be $h$-chromatically unique if $H$ is isomorphic to $H^{\prime}$ for every $h$-hypergraph $H^{\prime}$ such that $H^{\prime} \sim H$.

Non-trivial chromatically unique hypergraphs are extremely rare. One example of a non-trivial chromatically unique hypergraph was proposed by Borowiecki and Lazuka; it is $S H(n, 1, h)$. 
Theorem 2. [3] $S H(n, 1, h)$ is chromatically unique.

The proof of this result was completed in [11]. Note that for $p=h-1, S H(n, h-1, h)$ is an $h$-uniform hypertree. The chromaticity of $S H(n, p, h)$ may be stated as follows.

Theorem 3. [12] Let $n=h+(k-1) p$, where $h \geqslant 3, k \geqslant 1$ and $1 \leqslant p \leqslant h-1$. Then $S H(n, p, h)$ is h-chromatically unique for every $1 \leqslant p \leqslant h-2$; for $p=h-1 S H(n, h-1, h)$ is h-chromatically unique for $k=1$ or $k=2$ but it has not this property for $k \geqslant 3$. Moreover, $S H(n, p, h)$ is not chromatically unique for every $p, k \geqslant 2$.

$S H(n, p, h)$ is quasi-linear with $r=h-p$ and it is a path for $k=2$.

Since $P_{2}^{h, r}$ is a sunflower hypergraph $S H(n, p, h)$ with $p=h-r$ having $k=2$ edges, from Theorem 1.3 it follows that $P_{2}^{h, r}$ is $h$-chromatically unique for every $1 \leqslant r \leqslant h-1$. Also $P_{m}^{h, 1}$ is an $h$-uniform hypertree, hence for $m \geqslant 3$ it is not $h$-chromatically unique. We shall prove that $P_{m}^{h, r}$ for every $m \geqslant 1$ and $C_{m}^{h, r}$ for every $m \geqslant 3$ are $h$-chromatically unique hypergraphs in the set of quasi-linear hypergraphs provided $r \geqslant 2$ and $h \geqslant 3 r-1$. In [10] it was shown that $C_{m}^{h, r}$ is $h$-chromatically unique for $r=1$ and every $m, h \geqslant 3$, but it is not chromatically unique for $r=1$ and $m, h \geqslant 3[2]$. The chromaticity of non-uniform hypertrees was studied by Walter [15].

\section{Main results}

We need the following result about the first coefficients of the chromatic polynomial of a quasi-linear $h$-hypergraph with a particular structure relatively to subhypergraphs induced by 3 edges.

Lemma 4. Let $r \geqslant 2, h \geqslant 2 r+1$ and $H$ be a quasi-linear h-hypergraph of order $n$ and size $m$ having the property that all subhypergraphs induced by 3 edges have one of the following patterns:

a) $P_{3}^{h, r}$; b) $P_{2}^{h, r}$ and an isolated edge, or c) 3 isolated edges. Then

$$
P(H, \lambda)=\lambda^{n}-m \lambda^{n-h+1}+\beta_{1} \lambda^{n-2 h+r+1}+\beta_{2} \lambda^{n-2 h+2}-\beta_{3} \lambda^{n-3 h+2 r+1}+R(\lambda),
$$

where $R(\lambda)$ is a polynomial in $\lambda$ of degree at most equal to $n-3 h+2 r, \beta_{2}$ is the number of pairwise disjoint edges of $H$ and $\beta_{1}$ and $\beta_{3}$ are the numbers of induced subhypergraphs of $\mathrm{H}$ isomorphic to $P_{2}^{h, r}$ and $P_{3}^{h, r}$, respectively.

Proof. By the hypothesis we have $n-h+1>n-2 h+r+1>n-2 h+2>n-3 h+2 r+1$. If $W \subset E(H)$ in (1) consists of one edge we get $N(n-h+1, j)=m$ if $j=1$ and $N(n-h+1, j)=0$ otherwise.

If $|W|=2$ then $N(n-2 h+r+1,2)$ and $N(n-2 h+2,2)$ count the number of unordered pairs $\{E, F\}$ of edges such that $|E \cap F|=r$ and $E \cap F=\emptyset$, respectively. In these two cases suppose that there exists an edge $G \in \mathcal{E}, G \neq E, F$, such that $G \subset E \cup F$. Denote $i=|G \cap E \cap F|$. It follows that $0 \leqslant i \leqslant r,|G \cap(E \backslash F)|=r-i,|G \cap(F \backslash E)|=r-i$, thus yielding $h=|G|=2 r-i \leqslant 2 r$, which contradicts the hypothesis. It follows that 
$N(n-2 h+r+1, j)=N(n-2 h+2, j)=0$ for every $j \neq 2$ and $\beta_{1}, \beta_{2}$ represent the numbers of induced subhypergraphs of $H$ consisting of $P_{2}^{h, r}$ and of an unordered pair of disjoint edges, respectively.

Similarly, if $|W|=3$, by the hypoyhesis 3 edges can induce only subhypergraphs of types a), b) or c). We obtain that $N(n-3 h+2 r+1), N(n-3 h+r+2,3)$ and $N(n-3 h+3,3)$ count the subhypergraphs of $H$ induced by an unordered triple of edges $\{D, E, F\}$ such that these subhypergraphs are isomorphic to $P_{3}^{h, r}, P_{2}^{h, r}$ and an isolated edge and 3 isolated edges, respectively. We also have $n-3 h+2 r+1>n-3 h+r+2>n-3 h+3$ since $r \geqslant 2$ and $N(n-3 h+2 r+1, j)=0$ for every $0 \leqslant j \leqslant 2$.

If the edges $D, E, F$ of $H$ induce a $P_{3}^{h, r}$, where $D \cap F=\emptyset$, suppose that there exists an edge $G \neq D, E, F$ such that $G \subset D \cup E \cup F$. We have proved that $G \not \subset E \cup F$, thus implying $G \cap D \neq \emptyset$; similarly $G \not \subset D \cup F$ implies $G \cap E \neq \emptyset$. We have found 3 edges $D, E, G$ such that $D \cap E \neq \emptyset, D \cap G \neq \emptyset$ and $E \cap G \neq \emptyset$, which contradicts the hypothesis. This implies that $N(n-3 h+2 r+1, j)=0$ for every $j \geqslant 4$. Since by adding new edges to $W$ the number of components $c(W)$ decreases, it follows that $P(H, \lambda)$ is given by (4), where $\beta_{3}$ is the number of induced subhypergraphs of $H$ isomorphic to $P_{3}^{h, r}$.

Theorem 5. Let $H$ be an antilinear h-hypergraph such that $P(H, \lambda)=P(G, \lambda)$, where $G$ is $P_{m}^{h, r}(m \geqslant 1)$ or $C_{m}^{h, r}(m \geqslant 3)$. If $r \geqslant 2$ and $h \geqslant 3 r-1$ then $H$ is isomorphic to $G$.

Proof. It is trivial to see that $P_{m}^{h, r}$ for $1 \leqslant m \leqslant 3$ and $C_{3}^{h, r}$ are $h$-chromatically unique. Let $m \geqslant 4$. We shall consider two subcases:

I. $G=P_{m}^{h, r}$ and II. $G=C_{m}^{h, r}$.

I. Let $H$ be an antilinear $h$-hypergraph such that $P(H, \lambda)=P\left(P_{m}^{h, r}, \lambda\right)$.

The order of an hypergraph is being determined by the leading term of the chromatic polynomial, it follows that $H$ has order $n=h+(m-1)(h-r)$. From (2) one deduces that

$$
P\left(P_{m}^{h, r}, \lambda\right)=\lambda^{n}-m \lambda^{n-h+1}+\alpha_{1} \lambda^{n-2 h+r+1}+\alpha_{2} \lambda^{n-2 h+2}-\alpha_{3} \lambda^{n-3 h+2 r+1}+Q(\lambda),
$$

where $Q(\lambda)$ is a polynomial of degree at most equal to $n-3 h+r+2, \alpha_{1}=m-1$ is the number of subpaths $P_{2}^{h, r}$ of length two, $\alpha_{2}=\left(\begin{array}{c}m \\ 2\end{array}\right)-m+1$ is the number of pairs of pairwise disjoint edges and $\alpha_{3}=m-2$ is the number of subpaths $P_{3}^{h, r}$ of length three in $P_{m}^{h, r}$. Also since any spanning subhypergraph of $P_{m}^{h, r}$ induced by less than $m$ edges is not connected, it follows that in (5) the coefficient of $\lambda$ is $(-1)^{m}$, which implies that $H$ is also connected [15].

Since $H$ has all edges of cardinality $h$, it follows that the number of components of a spanning subhypergraph of $H$ may be $n, n-h+1$ or a smaller number. Any spanning subhypergraph of $H$ with $n$ vertices and $n-h+1$ components must contain only one edge. From (2) we deduce that $a_{n-h+1}=-N(n-h+1,1)=-|E(H)|$, hence $H$ has exactly $m$ edges. Every spanning subhypergraph of $H$ with $n$ vertices has two kinds of components: isolated vertices and components including at least $h$ vertices. The components including at least $h$ vertices will be called major components [10].

If such a spanning subhypergraph has at least two major components then it contains at most $n-2 h+2$ components and this bound is reached when the major components 
are two disjoint edges and minor components are $n-2 h$ isolated vertices. It follows that all coefficients $a_{n-h+1}, \ldots, a_{n-2 h+r+1}$ given by (2) correspond to the case when all spanning subhypergraphs of $H$ of order $n$ contain only one major component. In this way $N(n-h, j)$ counts the spanning subhypergraphs of $H$ consisting of a subset $Y$ of vertices (the major component) and $n-h-1$ isolated vertices, where $Y \subset V(H),|Y|=h+1$. Denote by $\varphi(Y)$ the number of edges included in $Y$. Because $Y$ induces a component having $h+1$ vertices, it follows that $\varphi(Y) \geqslant 2$ and for each $i \geqslant 2$ the union of any $i$ edges included in $Y$ equals $Y$. Since $N(n-h, 0)=N(n-h, 1)=0$, by (2) we get

$$
\begin{aligned}
& a_{n-h}=\sum_{j \geqslant 2}(-1)^{j} N(n-h, j)=\sum_{j \geqslant 2}(-1)^{j} \sum_{|Y|=h+1, \varphi(Y) \geqslant 2}\left(\begin{array}{c}
\varphi(Y) \\
j
\end{array}\right) \\
& =\sum_{|Y|=h+1, \varphi(Y) \geqslant 2} \sum_{j \geqslant 2}(-1)^{j}\left(\begin{array}{c}
\varphi(Y) \\
j
\end{array}\right)=\sum_{|Y|=h+1, \varphi(Y) \geqslant 2}(\varphi(Y)-1) .
\end{aligned}
$$

Since $a_{n-h}=0$ it follows that no such $Y$ can exist, or equivalently, for any two distinct edges $E, F$ we have $|E \cup F| \geqslant h+2$. If $Y \subset V(H),|Y|=h+2$ and $E, F \in E(H), E \neq F$ and $E, F \subset Y$ we get $E \cup F=Y$ since $|E \cup F| \geqslant h+2$. Since $a_{n-h-1}=0$ we deduce in the same way that $|E \cup F| \geqslant h+3$ and by induction we obtain that for any two distinct edges $E, F \in E(H)$ we have $|E \cup F| \geqslant 2 h-r$, or $|E \cap F| \leqslant r$.

Let now $Y \subset V(H),|Y|=2 h-r$ be a major component of a spanning subhypergraph of $H$ such that $Y$ contains exactly $j \geqslant 2$ edges. We shall prove that $j=2$. For this let $E, F \subset Y$ be two distinct edges such that $E \cup F=Y$. Suppose that there exists an edge $G, G \neq E, F$ such that $G \subset Y$. By denoting $a=|(E \backslash F) \cap G|$ and $b=|(F \backslash E) \cap G|$ we get $a+b \leqslant h$. Since $|G \cup E|=h+b \geqslant 2 h-r,|G \cup F|=h+a \geqslant 2 h-r$, it follows $a, b \geqslant h-r$, hence $a+b \geqslant 2 h-2 r$, which implies $h \geqslant 2 h-2 r$. But this contradicts the hypotheses $h \geqslant 3 r-1$ and $r \geqslant 2$. For hypergraph $H$ we can write

$$
\sum_{|Y|=2 h-r, \varphi(Y)=2} 1=a_{n-2 h+r+1}=m-1,
$$

which implies that $H$ contains exactly $m-1$ pairs of edges $\{E, F\}$ such that $|E \cap F|=r$, or $|E \cup F|=2 h-r$.

Let $p$ be such that $n-2 h+2<p<n-2 h+r+1$. If $Y \subset V(H),|Y|=n+1-p$ is a vertex subset inducing a major component of a spanning subhypergraph of $H$ it follows that $2 h-r<|Y|<2 h-1$. For every three distinct edges $E, F, G$ of $H$ we have

$$
|E \cup F \cup G| \geqslant|E|+|F|+|G|-|E \cap F|-|E \cap G|-|F \cap G| \geqslant 3 h-3 r,
$$

since every two edges have at most $r$ elements in common. But $3 h-3 r \geqslant 2 h-1$ since $h \geqslant 3 r-1$, which contradicts the property $|Y|<2 h-1$. Hence one has $\varphi(Y)=2$. This yields

$$
\sum_{|Y|=n+1-p, \varphi(Y)=2} 1=a_{p}=0 .
$$


It follows that no such $Y$ can exist, or for any two distinct edges $E, F$ we cannot have $2 h-r<|E \cup F|<2 h-1$, or $1<|E \cap F|<r$. But $H$ is antilinear, hence $|E \cap F| \neq 1$ and we have seen that $|E \cap F| \leqslant r$. It follows that $|E \cap F|=0$ or $r$, i.e., $H$ is also quasi-linear. Since $H$ has $m$ edges, is quasi-linear and connected, it may be obtained from $P_{2}^{h, r}$ by succesively adding $m-2$ distinct edges such that every new edge has $r$ vertices in common with at least one existing edge.

We will define two potential functions, $\alpha$ and $\beta$, for any $h$-uniform hypergraph $K$ of size $m: \alpha(K)=\alpha_{1}(K)-m$ and $\beta(K)=\alpha_{3}(K)-m$, where $\alpha_{1}(K)$ and $\alpha_{3}(K)$ are the numbers of induced subhypergraphs of $K$ isomorphic to $P_{2}^{h, r}$ and to $P_{3}^{h, r}$, respectively. We have deduced that for every $m \geqslant 1 \alpha\left(P_{m}^{h, r}\right)=\alpha(H)=-1$. If $K$ is an $h$-uniform quasi-linear hypergraph, then by adding a new edge $E \subset V(K), E \notin E(K)$ which intersects at least an edge from $E(K)$, we get a new hypergraph $K_{1}$ and $\alpha\left(K_{1}\right) \geqslant \alpha(K)$. Equality holds if and only if $E$ intersects exactly one edge from $E(K)$. Since $\alpha(H)=\alpha\left(P_{2}^{h, r}\right)=-1$, it follows that $H$ is obtained from $P_{2}^{h, r}$ by adding $m-2$ distinct edges such that every new edge has $r$ vertices in common with exactly one existing edge. This implies that every subhypergraph of $H$ induced by three edges has one of types a), b) or c). Since $P(H, \lambda)=P\left(P_{m}^{h, r}, \lambda\right)$, by Lemma 2.1 we obtain that $\alpha_{3}(H)=m-2=\alpha_{3}\left(P_{m}^{h, r}\right)$, hence $\beta(H)=\beta\left(P_{m}^{h, r}\right)=-2$. With the same notation as above we deduce $\beta\left(K_{1}\right) \geqslant \beta(K)$ and equality holds if and only if $E$ intersects exactly one edge which belongs to exactly one path $P_{2}^{h, r}$, which is an induced subhypergraph of $K$, unless $K_{1}$ is a sunflower hypergraph. Now the proof follows by induction: if we add a new edge to $P_{2}^{h, r}$ (having $\alpha\left(P_{2}^{h, r}\right)=-1$ ) such that potential function $\alpha$ remains unchanged, we get $P_{3}^{h, r}$. Let $i \geqslant 3$; if we add a new edge to $P_{i}^{h, r}$ such that both potential functions $\alpha$ and $\beta$ remain unchanged, this new edge must have $r$ vertices in common only with a terminal edge of $P_{i}^{h, r}$ and one obtains in this way the hypergraph $P_{i+1}^{h, r}$.

II. In the case of cycles $C_{m}^{h, r}$ with $m \geqslant 4$ we deduce as above that polynomial $P\left(C_{m}^{h, r}, \lambda\right)$ has $\alpha_{1}=m, \alpha_{2}=\left(\begin{array}{c}m \\ 2\end{array}\right)-m, \alpha_{3}=m$. If $H$ is antilinear and chromatically equivalent to $C_{m}^{h, r}$ then $H$ has order $m(h-r)$ and size $m$ and it is connected. As in the case of paths $P_{m}^{h, r}$ we deduce that $H$ has exactly $m$ unordered pairs of edges $\{E, F\}$ such that $|E \cap F|=r$ and $H$ is quasi-linear too. Also $H$ may be built from $P_{2}^{h, r}$ in $m-2$ steps, each step consisting in addition of a new edge $E$, having $r$ vertices in common with $t \geqslant 1$ existing edges $F_{1}, \ldots, F_{t}$, i.e., $\left|E \cap F_{1}\right|=\ldots=\left|E \cap F_{t}\right|=r$.

We have $\alpha\left(C_{m}^{h, r}\right)=\alpha(H)=0$, but $\alpha\left(P_{2}^{h, r}\right)=-1$. Since at each step potential function $\alpha$ increases or remains constant, it follows that in one step $\alpha$ increases by 1 and in $m-3$ steps it remains constant (equal to 0 or -1 ). It increases by 1 when the new edge $E$ intersects exactly two existing edges and remains constant when $E$ intersects exactly one existing edge. Suppose that $E$ intersects exactly two existing edges, $F_{1}$ and $F_{2}$, i.e., $\left|E \cap F_{1}\right|=\left|E \cap F_{2}\right|=r$ and $\left|F_{1} \cap F_{2}\right|=r$. We shall prove that this case is not possible, i.e., we must have $F_{1} \cap F_{2}=\emptyset$. Suppose that $\left|F_{1} \cap F_{2}\right|=r$ and denote $i=\left|E \cap F_{1} \cap F_{2}\right|$. It follows that $0 \leqslant i \leqslant r,\left|E \cap\left(F_{1} \backslash F_{2}\right)\right|=\left|E \cap\left(F_{2} \backslash F_{1}\right)\right|=r-i$. In this case $E$ contributes $h-2 r+i=\left|E \backslash\left(F_{1} \cup F_{2}\right)\right|$ new vertices. Since $P_{2}^{h, r}$ and $H$ have $2 h-r$ and $m(h-r)$ vertices respectively, and whenever $\alpha$ remains unchanged the new edge contributes $h-r$ new vertices $\left(m-3\right.$ times), we obtain that $i=0$, which means that $E, F_{1}, F_{2}$ induce a 
subhypergraph isomorphic to $C_{3}^{h, r}$.

In this case $H$ has the property that all subhypergraphs induced by 3 edges have the types a), b), c) and exactly one subhypergraph is isomorphic to $C_{3}^{h, r}$. A result similar to Lemma 2.1 also holds and the contribution of the spanning subhypergraph of $H$ consisting of $C_{3}^{h, r}$ and $n-3 h+3 r$ isolated vertices is $-\lambda^{n-3 h+3 r+1}$, which must be added to the polynomial given by (4). We have $n-3 h+3 r+1>n-3 h+2 r+1$ and $n-3 h+3 r+1<n-2 h+2$, unless $h=3 r-1$. If $n-3 h+3 r+1<n-2 h+2$ the monomial $-\lambda^{n-3 h+3 r+1}$ does not appear in $P\left(C_{m}^{h, r}, \lambda\right)$; if $h=3 r-1$ the coefficient of $\lambda^{n-2 h+2}$ equals $\alpha_{2}-1=\left(\begin{array}{c}m \\ 2\end{array}\right)-m-1$, a contradiction.

Consequently, $E$ intersects two existing edges $F_{1}, F_{2}$ such that $F_{1} \cap F_{2}=\emptyset$, which implies that $H$ contains an induced subhypergraph $H_{1}$ which is isomorphic to a cycle $C_{s}^{h, r}$ with $4 \leqslant s \leqslant m$. If $s=m$ then $H$ is isomorphic to $C_{m}^{h, r}$ and we are done. Otherwise, $H$ may be obtained from $C_{s}^{h, r}$ by succesively adding $m-s$ distinct edges such that every new edge has $r$ vertices in common with exactly one existing edge. We have $\beta\left(C_{s}^{h, r}\right)=0$; at the first step we get $\beta\left(C_{s}^{h, r}+E\right)=1$ if $E$ is such an edge. Since $\beta$ is increasing, we deduce $\beta(H) \geqslant 1$. But in this case every 3 edges of $H$ induce a subhypergraph of type a), b) or c), which implies that $\beta(H)=\beta_{3}-m=\alpha_{3}-m=0$, a contradiction.

Note that $P_{m}^{h, r}$ is not chromatically unique for any $m \geqslant 3, r \geqslant 1$ and $h \geqslant 2 r+1$, since any hypergraph containing a pendant path of length at least two is not chromatically unique [14].

Since every quasi-linear hypergraph is antilinear for every $r \geqslant 2$ we get:

Corollary 6. Let $r \geqslant 2, h \geqslant 3 r-1, m \geqslant 3$ and $H$ be a quasi-linear hypergraph such that $P(H, \lambda)=P(G, \lambda)$, where $G$ is $P_{m}^{h, r}$ or $C_{m}^{h, r}$. Then $H$ is isomorphic to $G$.

\section{Acknowledgements}

The author thanks the referee for helpful comments.

\section{References}

[1] C. Berge. Graphs and hypergraphs. North-Holland, Amsterdam, 1973.

[2] S. A. Bokhary, I. Tomescu, and A. A. Bhatti. On the chromaticity of multi-bridge hypergraphs. Graphs Combin., 25(2):145-152, 2009.

[3] M. Borowiecki and E. Eazuka. Chromatic polynomials of hypergraphs. Discuss. Math., Graph Theory, 20:293-301, 2000.

[4] C. Y. Chao and E. G. Whitehead, Jr.. On chromatic equivalence of graphs. In "Theory and Applications of Graphs" (Y. Alavi and D. R. Lick, Eds.), volume 642 of Lecture Notes in Math., pages 121-131. Springer, New York/Berlin, 1978.

[5] D. Dellamonica, V. Koubek, D. M. Martin, and V. Rödl. On a conjecture of Thomassen concerning subgraphs of large girth. J. Graph Theory, 67:316-331, 2011. 
[6] K. Dohmen. Chromatische Polynome von Graphen und Hypergraphen. Dissertation, Düsseldorf, 1993.

[7] P. Erdös and R. Rado. Intersection theorems for systems of sets. J. London Math. Soc., 35:85-90, 1960.

[8] Z. Füredi. On finite set-systems whose intersection is a kernel of a star. Discrete Math., 47(1):129-132, 1983.

[9] K. M. Koh and K. L. Teo. The search for chromatically unique graphs. Graphs Combin., 6:259-285, 1990.

[10] I. Tomescu. Chromatic coefficients of linear uniform hypergraphs. J. Combinatorial Theory Ser. B, 2(72):229-235, 1998.

[11] I. Tomescu. Sunflower hypergraphs are chromatically unique. Discrete Math., 285:355-357, 2004.

[12] I. Tomescu. On the chromaticity of sunflower hypergraphs $S H(n, p, h)$. Discrete Math., 307:781-786, 2007.

[13] I. Tomescu and S. Javed. On the chromaticity of quasi-linear hypergraphs (submitted).

[14] I. Tomescu. On chromaticity of hypergraphs with pendant paths (submitted).

[15] M. Walter. Some results on chromatic polynomials of hypergraphs. Electronic J. Combinatorics, 16, R94, 2009. 